\title{
CERTAIN INVERSION FORMULAS FOR THE LAPLACE TRANSFORM
}

DENNIS B. AMES

1. Introduction. In this paper certain inversion formulas are obtained for the Laplace transform. The case of a periodic determining function is first treated and some conclusions are reached about such functions when their transforms vanish at points in an arithmetical progression on a vertical line. This yields a contrast with Lerch's theorem. Later an inversion formula is derived for the more general case when the determining function is not assumed to be periodic but does have a finite abscissa of absolute convergence.

Let $F(q)$ be a real function of the real variable $q$ whose Laplace transform, $f(x+i y)=u(x, y)+i v(x, y)$, is convergent for $x>c$. Then

$$
\begin{aligned}
& u(x, y)=\int_{0}^{\infty} F(q) \cos y q e^{-x q} d q, \\
& v(x, y)=-\int_{0}^{\infty} F(q) \sin y q e^{-x q} d q .
\end{aligned}
$$

If the transform of $F(q)$ is known, then (1) give the real transforms of $F(q) \cos y q$ and $F(q) \sin y q$. Define $\phi(x, t)=\int_{0}^{t} F(q) e^{-x q} d q$ for $t>0$ and $\phi(x, 0)=0$. Then $\phi(x, t)$ is a continuous function of $t$ for $t \geqq 0$.

2. Heuristic treatment of the periodic case. Let $F(q)$ be periodic with the period $2 k$. Then for $x>0$

$$
\begin{aligned}
\left(1-e^{-2 k x}\right) f(x) & =\int_{0}^{2 k} F(q) e^{-x q} d q, \\
\left(1-e^{-2 k x}\right) u\left(x, \frac{n \pi}{k}\right) & =\int_{0}^{2 k} F(q) \cos \frac{n \pi q}{k} e^{-x q} d q, \\
\left(1-e^{-2 k x}\right) v\left(x, \frac{n \pi}{k}\right) & =-\int_{0}^{2 k} F(q) \sin \frac{n \pi q}{k} e^{-x q} d q .
\end{aligned}
$$

Let $a_{0} / 2+\sum_{n=1}^{\infty}\left(a_{n} \cos (n \pi t / k)+b_{n} \sin (n \pi t / k)\right)$ be the Fourier series of the function of period $2 k$ which is equal to $\phi(x, t)$ for $0<t$ $<2 k$. If inversion of the order of integration is permissible then we have

Presented to the Society, February 26, 1949; received by the editors September 7, 1948 and, in revised form, October 19, 1948. 


$$
\begin{aligned}
\frac{a_{0}}{2} & =\frac{1}{2 k} \int_{0}^{2 k} d t \int_{0}^{t} F(q) e^{-x q} d q \\
& =\frac{1}{2 k} \int_{0}^{2 k} F(q) e^{-x q} d q \int_{0}^{2 k} d t \\
& =f(x)+\frac{1}{2 k}\left(1-e^{-2 k x}\right) f^{\prime}(x) . \\
a_{n} & =\frac{1}{k} \int_{0}^{2 k} \cos \frac{n \pi t}{k} d t \int_{0}^{t} F(q) e^{-x q} d q \\
& =\frac{1}{k} \int_{0}^{2 k} F(q) e^{-x q} d q \int_{0}^{2 k} \cos \frac{n \pi t}{k} d t \\
& =\frac{1}{n \pi}\left(1-e^{-2 k x}\right) v\left(x, \frac{n \pi}{k}\right),
\end{aligned}
$$

and similarly

$$
b_{n}=\frac{1}{n \pi}\left[1-e^{-2 k x}\right]\left[u\left(x, \frac{n \pi}{k}\right)-f(x)\right] .
$$

Thus if the function $\phi(x, t)$ satisfies any set of conditions which ensure the convergence of its Fourier series and its representation by this series, then we would formally obtain for $x>0$ and $0<t<2 k$ the following inversion formula

$$
\begin{aligned}
\int_{0}^{t} F(q) e^{-x q} d q=f(x) & +\frac{1}{2 k}\left(1-e^{-2 k x}\right) f^{\prime}(x) \\
& +\frac{1}{\pi}\left(1-e^{-2 k x}\right) \sum_{n=1}^{\infty} \frac{1}{n}\left\{v\left(x, \frac{n \pi}{k}\right) \cos \frac{n \pi t}{k}\right. \\
& \left.+\left[u\left(x, \frac{n \pi}{k}\right)-f(x)\right] \sin \frac{n \pi t}{k}\right\} .
\end{aligned}
$$

3. Proof of the inversion formula for the periodic case. The foregoing heuristic argument suggests then a study of the expression

$$
\begin{aligned}
K=\frac{1}{\pi}\left(1-e^{-2 k x}\right) \sum_{n=1}^{\infty} \frac{1}{n}\left\{v\left(x, \frac{n \pi}{k}\right)\right. & \cos \frac{n \pi t}{k} \\
& \left.+\left[u\left(x, \frac{n \pi}{k}\right)-f(x)\right] \sin \frac{n \pi t}{k}\right\}
\end{aligned}
$$

for $x>0$ and $0<t<2 k$. 
Call $H=f(x)+(1 / 2 k)\left(1-e^{-2 k x}\right) f^{\prime}(x)$. By use of $(2)$ it is seen that

$$
K=\frac{1}{\pi} \sum_{n=1}^{\infty} \int_{0}^{2 k} F(q) e^{-x q} d q \frac{1}{n}\left[\sin \frac{n \pi(t-q)}{k}-\sin \frac{n \pi t}{k}\right]
$$

with $x>0$ and $0<t<2 k$.

Now the series $\sum_{n=1}^{\infty}(1 / n \pi) \sin (n \pi \theta / k)$ is uniformly convergent to $(1 / 2 k)(k-\theta)$ for $0<\theta<2 k$ and is boundedly convergent in $0 \leqq \theta$ $\leqq 2 k$. In fact the partial sums are less than or equal to $1 / \pi+1 / 2$ for all $n$ and all $\theta$. Hence inversion of summation and integration is valid. This is true when the factor $F(q) e^{-x q}$ is integrable but not necessarily bounded. If we write $K$ as

$$
\begin{aligned}
K= & \int_{0}^{t} F(q) e^{-x q} d q \sum_{n=1}^{\infty} \frac{1}{n \pi}\left[\sin \frac{n \pi(t-q)}{k}-\sin \frac{n \pi t}{k}\right] \\
& -\int_{t}^{2 k} F(q) e^{-x q} d q \sum_{n=1}^{\infty} \frac{1}{n \pi}\left[\sin \frac{n \pi(q-t)}{k}+\sin \frac{n \pi t}{k}\right],
\end{aligned}
$$

it is seen that the first sum on the right is $q / 2 k, 0<q<t<2 k$, while the second sum is $(1 / 2 k)(2 k-q), 0<t<q<2 k$. Hence

$$
\begin{aligned}
K & =\frac{1}{2 k} \int_{0}^{2 k} q F(q) e^{-x q} d q-\int_{t}^{2 k} F(q) e^{-x q} d q \\
& =\frac{-1}{2 k} \frac{d}{d x}\left[\left(1-e^{-2 k x}\right) f(x)\right]-\int_{t}^{2 k} F(q) e^{-x q} d q .
\end{aligned}
$$

Thus $H+K=\int_{0}^{t} F(q) e^{-x q} d q$. Hence we have proved:

TheOREM I. Let $F(q)$ have a Laplace transform given by $f(x+i y)$ $=u(x, y)+i v(x, y)$. Let $F(q)$ have the period $2 k$. Then for $x>0$ and $0<t<2 k$, the inversion formula (3) is true.

The formula (3) can be written

$$
\begin{aligned}
& \int_{0}^{t} F(q) e^{-x q} d q \\
& =f(x)+\left\{1-e^{-2 k x}\right\}\left\{\frac{1}{2 k} f^{\prime}(x)-\frac{1}{2 k}(k-t) f(x)\right. \\
& \left.+\frac{1}{\pi} \sum_{n=1}^{\infty} \frac{1}{n}\left[v\left(x, \frac{n \pi}{k}\right) \cos \frac{n \pi t}{k}+u\left(x, \frac{n \pi}{k}\right) \sin \frac{n \pi t}{k}\right]\right\}
\end{aligned}
$$

for $0<t<2 k$.

4. A second inversion formula. Consider the expression 


$$
\begin{aligned}
W=\frac{1}{k}\left\{1-e^{-2 k x}\right\}\left\{\frac{f(x)}{2}+\sum_{n=1}^{\infty}\left[u\left(x, \frac{n \pi}{k}\right)\right.\right. & \cos \frac{n \pi t}{k} \\
& \left.\left.-v\left(x, \frac{n \pi}{k}\right) \sin \frac{n \pi t}{k}\right]\right\} .
\end{aligned}
$$

This expression is obtained formally by differentiating the right side of (3) with respect to $t$. Let $F(q)$ be of period $2 k$. Then by use of (2) it can be seen that $W$ is the Fourier series of the function given by $G(t)=F(t) e^{-x t}, 0<t<2 k, G(t+2 k)=G(t)$. We thus have:

THEOREM II. Let $F(q)$ have a Laplace transform given by $f(x+i y)$ $=u(x, y)+i v(x, y)$. Let it have the period $2 k$. If the function $F(t) e^{-x t}$, $x>0,0<t<2 k$, satisfies any set of conditions which ensure the convergence of its Fourier series to the function, then the following inversion formula is true:

$$
\begin{aligned}
F(t)=\frac{1}{k} e^{x t}\left[1-e^{-2 k x}\right]\left[\frac{1}{2} f(x)+\sum_{n=1}^{\infty}\right. & \left(u\left(x, \frac{n \pi}{k}\right) \cos \frac{n \pi t}{k}\right. \\
& \left.\left.-v\left(x, \frac{n \pi}{k}\right) \sin \frac{n \pi t}{k}\right)\right]
\end{aligned}
$$

for $0<t<2 k$. If $F(t)$ is of bounded variation in the neighborhood of the point $t$, then the $F(t)$ on the left of the formula represents $(F(t+0)$ $+F(t-0)) / 2$.

If $F(t)$ is complex, $F(t)=F_{1}(t)+i F_{2}(t)$, then it is easy to see that an inversion formula similar to (4) exists, since (4) exists for each of $F_{1}$ and $F_{2}$. For $F_{1}$ and $F_{2}$ have individually the period $2 k$ if $2 k$ is real. If $F(t)$ is a nonperiodic function which is zero for $t>t_{0}$, then an inversion formula similar to (4) exists. The modifications are to suppress the factor $1-e^{-2 k x}$ and replace $2 k$ by $t_{0}$.

5. Some properties of the periodic case. Let $F(t)$ be real and have the real period $2 k$. Let its transform for some $x>0$ vanish at the points $x+i n \pi / k(n=1,2,3, \cdots)$ of a vertical line. Then $u(x, n \pi / k)$ and $v(x, n \pi / k)$ are zero and it is seen by (3a) that

$$
F(t)=\frac{1}{2 k}\left(1-e^{-2 k x}\right) f(x) e^{x t}, \quad 0<t<2 k .
$$

For a given transform $f(x+i y)$ this is the unique real periodic function. Of course a periodic function of period $2 k$ of the form $G(x) e^{x t}$, $0<t<2 k$, where $G$ is arbitrary would have a transform which vanishes at the same set of points. Since $u(x, y)$ and $v(x, y)$ are respectively 
even and odd functions of $y$ the transform would also vanish at the points where $n$ is a negative integer of the same vertical line.

If in addition the transform vanishes at the point $x$ on the real axis then it is seen that $F(t)=0$. Hence:

Theorem. A necessary and sufficient condition that the periodic function $F(t)$ of period $2 k$ be null is that its transform vanish at the points $x+i n \pi / k(n=0,1,2, \cdots)$ of a vertical line.

This theorem is true when $F$ is complex, as can be seen at once when the inversion formula for this case is set up as described in the previous section. Moreover if the transform vanishes only at the points $x+i n \pi / k(n=1,2,3, \cdots)$, then $F(t)$ is again given by (5).

More generally let $F(t)=F_{1}+i F_{2}$ and have the real period $2 k$. Let its transform $f$ vanish at the points $x+i y+i n \pi / k \quad(n=0, \pm 1$, $\pm 2, \cdots)$ of a vertical line where $y$ is not necessarily zero. Separating $f(x+i y+i n \pi / k)=0$ into real and imaginary parts we have

$$
\begin{aligned}
& \int_{0}^{2 k} e^{-x q}\left[F_{1} \cos \left(y+\frac{n \pi}{k}\right) q+F_{2} \sin \left(y+\frac{n \pi}{k}\right) q\right] d q=0, \\
& \int_{0}^{2 k} e^{-x q}\left[F_{2} \cos \left(y+\frac{n \pi}{k}\right) q-F_{1} \sin \left(y+\frac{n \pi}{k}\right) q\right] d \Psi=0 .
\end{aligned}
$$

Writing $-n$ for $n$ in each of these formulas and adding and subtracting the results for each formula, we obtain

$$
\begin{aligned}
& \int_{0}^{2 k} e^{-x q}\left[F_{2} \sin y q+F_{1} \cos y q\right] \cos \frac{\sin \frac{n \pi q}{k}}{k} d q=0, \\
& \int_{0}^{2 k} e^{-x q}\left[F_{2} \cos y q-F_{1} \sin y q\right] \sin \frac{n \pi q}{k} d q=0,
\end{aligned}
$$

$n=0, \pm 1, \pm 2, \cdots$. That $n=0$ is included is seen from the previous pair of equations. Hence if $F$ is bounded and has at most a finite number of discontinuities in the interval $0<q<2 k$, we obtain at each point of continuity

$$
F_{2} \sin y q+F_{1} \cos y q=0, F_{2} \cos y q-F_{1} \sin y q=0 .
$$

The determinant equals -1 , hence $F_{1}=0$ and $F_{2}=0$ at each point of continuity. Hence $F$ is a null function. We thus have the following theorem true whether $F$ is complex or real.

Theorem. A necessary and sufficient condition that a periodic function of period $2 k$ be null is that its transform vanish at the points $x+i y$ $+i n \pi / k(n=0, \pm 1, \pm 2, \cdots)$ of a vertical line. 
It is seen that the theorem is true if the common distance of the equidistant points on the vertical line has the form $\pi / m k$ where $m$ is an integer. The following example shows that if this distance does not have this form, then $F(t)$ is not necessarily null. Let $F=-1$, $0<t<k ; F=e^{k}, k<t<2 k ; F(t+2 k)=F(t)$. The transform of this function is $\left(e^{k}-e^{k s}\right) / s\left(1+e^{k s}\right)$. It vanishes at the points $s=1$ $+2 n \pi i / k(n=0, \pm 1, \pm 2, \cdots)$. Here the common distance on the vertical line $x=1$ is $2 \pi / k$.

6. Proof of an inversion formula for the general case. Let $F(q)$ have a Laplace transform which is absolutely convergent for $x>c$. It is of interest to see that the foregoing results lead to an inversion formula for this case where $F$ is not assumed periodic. The formula has a form similar to that of the periodic case. The fact that $H+K$ in $\S 3$ is independent of $k$ leads us quite naturally to a consideration of the expression $W$, defined below, as $k \rightarrow \infty$. For a fixed positive $t$ and for $x>c$ set up the following expression in which $k$ has any value for which $0<t<2 k$,

$$
\begin{aligned}
W & =\sum_{n=1}^{\infty} \frac{1}{n \pi}\left[v\left(x, \frac{n \pi}{k}\right) \cos \frac{n \pi t}{k}+u\left(x, \frac{n \pi}{k}\right) \sin \frac{n \pi t}{k}\right] \\
& =\sum_{n=1}^{\infty} \frac{1}{n \pi} \int_{0}^{\infty} F(q) e^{-x q} \sin \frac{n \pi(t-q)}{k} d q .
\end{aligned}
$$

Decompose the integral into three integrals: $W_{1}$ from 0 to $t, W_{2}$ from $t$ to $2 k$, and $W_{3}$ from $2 k$ to $\infty$, so that

$$
W=\sum_{n=1}^{\infty} \frac{1}{n \pi}\left(W_{1}+W_{2}+W_{3}\right) .
$$

From the discussion in $\$ 3$ we see that

$$
\begin{aligned}
& \sum_{n=1}^{\infty} \frac{1}{n \pi} W_{1}=\frac{1}{2 k} \int_{0}^{t} F(q) e^{-x q}(k-t+q) d q, \\
& \sum_{n=1}^{\infty} \frac{1}{n \pi} W_{2}=\frac{-1}{2 k} \int_{t}^{2 k} F(q) e^{-x q}(k-q+t) d q .
\end{aligned}
$$

Hence as $k \rightarrow \infty$ the limits of these are

$$
\frac{1}{2} \int_{0}^{t} F(q) e^{-x q} d q \text { and } \frac{-1}{2} \int_{t}^{\infty} F(q) e^{-x q} d q
$$

respectively. We now show that $\lim _{k \rightarrow \infty} \sum_{n=1}^{\infty} 1 / n \pi W_{8}=0$. 


$$
\sum_{n=1}^{\infty} \frac{1}{n \pi} W_{3}=-\sum_{n=1}^{\infty} \frac{1}{n \pi} \int_{2 k}^{\infty} F(q) e^{-x q} \sin \frac{n \pi(q-t)}{k} d q .
$$

Decompose the interval $(2 k, \infty)$ into the subintervals $(2 k$, $2 k+t), \quad(2 k+t, \quad 4 k+t), \cdots, \quad(2 m k+t, \quad 2 m k+2 k+t), \cdots$ where $m=1,2,3, \ldots$. In the subinterval $(2 k, 2 k+t)$ we have $0<2 k-t$ $<q-t<2 k$, while in $(2 m k+t, 2 m k+2 k+t)$ we have $2 m k<q-t$ $<2 m k+2 k$. Now

$$
\sum_{n=1}^{\infty} \frac{1}{n \pi} \sin \frac{n \pi \theta}{k}=\frac{1}{2 k}[(2 m+1) k-\theta], \quad 2 m k<\theta<2(m+1) k .
$$

In each subinterval the series involved is boundedly convergent and uniformly convergent in any closed interval within the integration limits. Hence the operations of summation and integration are commutable. It is seen then that

$$
\begin{aligned}
\sum_{n=1}^{\infty} \frac{1}{n \pi} W_{3}= & \frac{1}{2 k} \int_{2 k}^{\infty}(t-q) F(q) e^{-x q} d q+\frac{1}{2} \int_{2 k}^{2 k+t} F(q) e^{-x q} d q \\
& +\frac{1}{2} \sum_{m=1}^{\infty}(2 m+1) \int_{2 m k+t}^{2(m+1) k+t} F(q) e^{-x q} d q .
\end{aligned}
$$

The first two terms on the right have limits of zero as $k \rightarrow \infty$. Consider the last term. Now for $q$ sufficiently large, $|F(q)|<A e^{x_{0} q}$ for $x_{0}>c$. For a given $x>c$ choose an $x_{0}$ such that $x>x_{0}>c$. We have then for sufficiently large $k$, calling $x-x_{0}=p>0$, and designating this last term by $T$,

$$
\begin{aligned}
|T| & <\frac{A e^{-p t}}{2 p}\left(1-e^{-2 k p}\right) \sum_{m=1}^{\infty}(2 m+1) e^{-2 m k p} \\
& =\frac{A e^{-p t}\left(3-e^{-2 k p}\right)}{2 p\left(e^{2 k p}-1\right)} .
\end{aligned}
$$

Hence $T$ has a limit of zero as $k \rightarrow \infty$. Combining our results we have

$$
\begin{aligned}
\lim _{t \rightarrow \infty} W & =\frac{1}{2} \int_{0}^{t} F(q) e^{-x q} d q-\frac{1}{2} \int_{t}^{\infty} F(q) e^{-x q} d q \\
& =\int_{0}^{t} F(q) e^{-x q} d q-\frac{1}{2} f(x) .
\end{aligned}
$$

We have proved:

Theorem. Let $F(q)$ be real and let its Laplace transform $f(x+i y)$ 
$=u(x, y)+i v(x, y)$ be absolutely convergent for $x>c$. Then for $x>c$ and $t>0$

$$
\begin{aligned}
& \int_{0}^{t} F(q) e^{-x} d q=\frac{1}{2} f(x)+\lim _{k \rightarrow \infty} \sum_{n=1}^{\infty} \frac{1}{n \pi} {\left[u\left(x, \frac{n \pi}{k}\right) \sin \frac{n \pi t}{k}\right.} \\
&\left.+v\left(x, \frac{n \pi}{k}\right) \cos \frac{n \pi t}{k}\right] .
\end{aligned}
$$

As an example of the formula consider $f(s)=1 /(s+1)$ and take $x=0$. We obtain

$$
\int_{0}^{t} F(q) d q=\frac{1}{2}+\lim _{k \rightarrow \infty} \sum_{n=1}^{\infty}\left[\frac{k^{2} \sin n \pi t / k}{n \pi\left(k^{2}+n^{2} \pi^{2}\right)}-\frac{k \cos n \pi t / k}{\left(k^{2}+n^{2} \pi^{2}\right)}\right] .
$$

For any $t$, provided $k$ is large enough, we have for the sum of the sine series $[(1-t / k)-\sinh (k-t) / \sinh k] / 2$. Its limit is $\left(1-e^{-t}\right) / 2$. The sum of the cosine series is $[\cosh (k-t) / \sinh k-1 / k] / 2$. Its limit is $e^{-t} / 2$. Hence $\int_{0}^{t} F(q) d q=1-e^{-t}$ and $F(t)=e^{-t}$. In the proof of the above theorem the following hypotheses suffice for its truth:

(i) $F$ is real and integrable, $t \geqq 0$. (It is not assumed bounded.)

(ii) $\int_{0}^{\infty} F e^{-s t} d t$ is absolutely convergent, Real $s=x>c$.

Hence with no implication that the transform is analytic it follows from (6) that if the transform vanishes on a vertical line in the halfplane Real $s>c$ then $F$ is null and the transform is identically zero.

Rensselaer Polytechnic Institute 